\title{
La sinagoga del Sofer en Toledo
}

\author{
Jean PASSINI ${ }^{*}$ \\ C.N.R.S., Lyon
}

En su libro sobre las sinagogas españolas ${ }^{1}$, Francisco Cantera Burgos consagra un capítulo a las de Toledo. A las diez enumeradas en 1391 en el poema de Albené, añade dos mencionadas en documentos de fines del siglo XIV y comienzos del XV, una de ellas llamada del Sofer, cuyo estudio continuará once años después ${ }^{2}$, y otra situada en el barrio de Caleros ${ }^{3}$. Propone ubicar la del Sofer junto al barrio de los tintoreros, no lejos del Alcaná, frente a la puerta principal de la catedral ${ }^{4}$, en la judería vieja, sin llegar a precisar los límites de este barrio. La puesta al día de documentos ${ }^{5}$ del monasterio de Santo Domingo el Real y del hospital de la Misericordia nos permite precisar el emplazamiento de la sinagoga del Sofer. Ésta, mencionada desde 1397 a 1480 en documentos notariales o en relaciones de censos relativas a las casas vecinas conocidas como de la Higuera y de la

* jpassini@free.fr

Agradecemos a D. García la traducción al castellano de este artículo.

1 F. Cantera Burgos, Sinagogas españolas (Madrid 1955).

2 F. Cantera Burgos, «Relieves históricos de la judería de Toledo», Sefarad 26 (1966) págs. 20-322.

3 F. CAntera Burgos, Sinagogas españolas, págs. 52-55.

4 Esta conclusión ha sido adoptada por Porres y tras él por numerosos autores; véase J. Porres, Historia de la calles de Toledo, I ( $3^{\mathrm{a}}$ ed. Toledo 1988; $4^{\mathrm{a}}$ ed. Toledo 2002).

5 Abreviaturas utilizadas: A.D.P.T., Archivo de la Diputación Provincial de Toledo; A.H.N Archivo Histórico Nacional; A.H.P.T., Archivo Histórico Provincial de Toledo; A.S.D.R., Archivo de Santo Domingo el Real.

Sefarad 64 (2004) págs. 141-157

(C) CSIC

ISSN 037-0894 
Atahona, no figura en ellos después de 1480. Si podemos situar con precisión la casa de la Higuera, sobre la que el monasterio de Santo Domingo el Real percibía aún en la segunda mitad del siglo XVIII un tributo de 200 maravedís, tendremos localizado el lugar donde se alzaba la sinagoga del Sofer. Tal es la finalidad de este artículo, al cual se añade una reflexión acerca del barrio judío en el siglo XV.

\section{DATOS TEXTUALES}

En 1397, la casa de la Higuera es objeto de venta judicial a causa de una deuda de 993 maravedís que su propietario, don Çuleman Jarada, dejó pendiente al abandonar Toledo en $1391^{6}$. Esta casa, situada «en la que solia ser juderia, cerca de los tintores», se levantaba entre una casa de don Mose Izrael y la sinagoga «que dizen del Sofer». La venta está registrada en un documento de $1402^{7}$ que concreta la situación de la casa de la Higuera «en la que fue juderia», junto a una casa «que dicen de la Atahona ${ }^{8}$ » y la sinagoga del Sofer. Un año después, otro documento nos recuerda que don Çulemán poseía en la judería, «en la calle que llaman Abendonja», una casa llamada de la Higuera, entre la «atahona» de don Alvar Pérez y la sinagoga del Sofer ${ }^{9}$.

6 F. CANTERA Burgos, Sinagogas españolas, edita por extenso uno de los documentos del monasterio de Santo Domingo el Real que había publicado resumido F. BAER, Die Juden im christlichen Spanien, I/2. Kastilien Inquisitionsakten (Berlin 1936) n..$^{\circ} 262$, págs. 245-246.

7 A.H.N., Clero, pergs., carp. 3078, n. $^{\circ} 2$, resumido por P. LEÓN TELLO, Judíos de Toledo, II. Inventario cronológico de documentos (Madrid 1979) pág. 203, n. ${ }^{\circ}$ 683: en 1402 (el 13 de febrero), «Diego Gonzalez Aben Çum tejedor de seda, y su mujer Leona diaz, reconocen que Pedro Lopez, commo tutor de Leonor y Mencia, hijos de Juan Rodriguez, les habia vendido una casa de los menores en Toledo en la que fue juderia desta dicha cibdad, las quales dizen de la Figuera, que se tiene con casas que dicen del Atahona e con la sinoga que dizen del sofer, las cuales eran en suelos de soror doña maria, hija del rey don Pedro».

8 La atahona era un molino movido por fuerza animal: «... Diego de Urrea le da su terminacion arabiga tahonetum, del verbo tahane que significa moler, pero mas cierge tahan, molere, contundere», S. DE COVARRUBIAS, Tesoro de la lengua castellana o española (Barcelona 1943)

9 Toledo, Museo Arqueológico, transcrito y publicado por F. CANTERA BuRGOS, «Relieves históricos de la judería de Toledo», págs. 305-322. Resumido en P. LEÓN TELLO, Judíos de Toledo, II, n. ${ }^{\circ}$ 686, pág. 204. 
Esta sinagoga aparece además en un documento de 1446 que recoge la transmisión a Martín de Guzmán del tributo percibido por doña Mencía Fernández sobre la casa que ésta poseía en la parroquia de Santo Tomé ${ }^{10}$. Dicho inmueble estaba junto a la casa de la Higuera de Luis Alfón, mayordomo de Santo Domingo, y las «casas de la xinoga que llaman el Sofer». Un año después, Martín de Guzmán vende al hospital de la Misericordia ${ }^{11}$ este tributo pagadero sobre las casas «que lindaban con la sinagoga que disen del Sofer y casas que fueron de Lyuis Alfon (casa de la Higuera) y luego, de Sancho Gonzalez, escribano». El mismo año, este Sancho González reconoce el censo que su padre Luis Alfón pagaba a doña Mencía Fernández y se declara dispuesto a pagarlo al hospital de la Misericordia ${ }^{12}$.

Por su parte, el libro de las medidas del hospital de la Misericordia ${ }^{13}$ señala que esta institución compra a Martín de Guzmán en 1447 la casa situada en la parroquia de Santo Tomé, «fondon de la puerta de la juderia de parte de fuera». Esta casa se alza en «la calle real», entre la casa «de la Figuera que son del dicho luys alfon mayordomo» $y$ «la xinoga que dicen del Sofer y la otra casa de los he-

${ }^{10}$ A.D.P.T., Toledo, Diputación Provincial, sig. antigua, cajón 2, leg. 2, n. ${ }^{\circ} 33$, resumido por P. LEÓn Tello, Judíos de Toledo, II, n. ${ }^{\circ} 832$. En 1446 (el 21 de junio), «Mencía Fernández traspasa a Martín de Guzmán, hijo del alguacil mayor de Sevilla Alvar Pérez de Guzmán su sobrino, el censo que el mayordomo de Santo Domingo el Real tenía que pagarle por unas casas que doña Mençía poseía en Toledo, colación de Santo Tomé, y lindaban con casas de Luis Alfón, mayordomo de Santo Domingo, casas de la Higuera y casas de la xinoga que llaman del çofer».

11 A.D.P.T., sig. antigua, cajón 2, leg. 2, resumido por P. LEÓN TELlo, Judíos de Toledo, II, pág. 254, n. ${ }^{\circ}$ 838: En 1447, «Martín Guzmán vende al Hospital de la Misericordia las casas que le había traspasado su tía Mencía Fernández, en Toledo, colacion de Santo Tome, y lindaban con la sinoga que disen de Sofer y con casas que fueron de Luis Alfon y luego, de Sancho Gonzalez, escribano».

12 A.D.P.T., sig. antigua, cajón 2, leg. 2, resumido por P. LEón TELlo, Judíos de Toledo, II, n. ${ }^{\circ}$ 837: En 1447 (el 22 de mayo) «Sancho González, escribano, reconoce el censo que había de pagar su padre Luis Alfón a doña Mencía Fernández, sobre unas casas lindantes con la sinagoga del Sofer, y se obliga a pagar el mismo censo al hospital de la Misericordia.»

13 A.D.P.T., Hospital de la Misericordia, libro 1, 1455. 
rederos de fernando gonzalez ${ }^{14} \gg$. Se compone de cinco cámaras ${ }^{15}$, una sobre la puerta de la casa, otra sobre la casa contigua, y una tercera que abre a la calle (Figura 1). El contenido de un documento del monasterio de Santo Domingo el Real, con fecha de 1460, nos hace pensar que la casa sobre la que Sancho González pagaba un tributo de 260 maravedís al hospital de la Misericordia no es otra que la Atahona, pegada a la casa de la Higuera ${ }^{16}$. La Atahona no figura en los reconocimientos de censos del hospital de la Misericordia más allá de $1509^{17}$.

Por otro lado, el monasterio de Santo Domingo el Real percibía en 1460 un tributo de 200 maravedís sobre la casa que tenía Sancho González, hijo de Luis Alfón ${ }^{18}$, en la parroquia de Santo Tomé, «a los tintores cerca la puerta de la juderia». Esta casa se situaba junto a «casas de Luys Gonçalez mayordomo que fue de Johan Gudiel e de la otra parte casas de Johan Gonçales Franco alguacil e de la otra parte casas que disen de la tahona que son del dicho Sancho Gonçales tributarias al ospytal de la misericordia.»

Un documento del Becerro de Santo Domingo el Real, no datado con precisión pero posterior a $1477^{19}$, atestigua que dicho monaste-

14 A.D.P.T., Inventario de los bienes del hospital de la Misericordia, libro 139.

15 A.D.P:T., Hospital de la Misericordia, libro 1, op. cit.: «Otrossi tiene e possee el dicho hospital un tributo de quantia de doscientos e sesenta maravedis en cada uno año en unas casas que tiene sancho gonçales fijo de luys alfonso que son fondon de la puerta de la Juderia de parte de fuera en las quales ay frontero de la puerta principal una camara sobre una casa de ferrando de la camara en que ay quatro varas e media en ancho e en luengo en esquadra.

Dentro de esta casa otra camara en que ay quatro varas en largo e tres varas e media en ancho.

Una camara sobre la puerta en que ay tres varas e media en largo e dos varas e media en ancho.

Otra camara en que ay nueve varas en largo e quatro varas e media en ancho.

Otra camara en que ay ocho varas en largo e quatro en ancho e tiene una puerta que sale a la calle.»

${ }^{16}$ A.H.N., Clero 15 118, Santo Domingo el Real, Libro de las medidas, pág. 32.

17 A.D.P:T., Hospital de la Misericordia, leg. 5, n. ${ }^{\circ}$ 12, 1443-1509.

18 A.H.N., Clero 15 118, Santo Domingo el Real, Libro de las medidas, pág. 32.

19 Este documento es posterior a 1477, año de adquisición de las casas en cuyo solar se edificaría el claustro del convento franciscano de San Juan de los Reyes. Bastantes de ellas pertenecían al regidor de Madrid Pedro Núñez de Toledo. Véase 
rio recibe del procurador Sancho González un tributo de 200 maravedís sobre la casa de la hija del rey, doña María de Toledo, situada en la parroquia de Santo Tomé «cerca de la puerta de la juderia ${ }^{20}$ a la mano izquierda como entran por la puerta de la juderia cerca del monasterio de San Juan de los Reyes». Esta casa, llamada «casas de la higuera», se alzaba junto a otra llamada «de la tahona», «tributaria al hospital de la misericordia». Otro documento de reconocimiento de un censo de 200 maravedís al monasterio de Santo Domingo el Real por Juan de Jarada, que ocupaba la casa de la Higuera, con fecha de 1507, señala que la puerta principal de ésta da a un adarve próximo a San Juan de los Reyes ${ }^{21}$. Los reconocimientos sucesivos de un censo de 200 maravedís a lo largo del siglo XVI ${ }^{22}$ no aportan ninguna información suplementaria sobre la

sobre este particular R. Del Cerro Malagón, M. J. Sainz, C. Delgado Valero, T. PÉrez Higueras, M. A. Franco Mata, et al., Arquitecturas de Toledo. Del periodo romano al gótico (Toledo 1992).

${ }^{20}$ A.S.D.R., Asiento de todas las posesiones del monasterio de Santo Domingo el Real, comenzado en 1507, fol. CCCCLXXIIIr, CD-Rom, pág. 363: «Tiene una casa en esta dicha ciudad de Toledo en la colacion de St Tome cerca de la puerta de la juderia y tiene las puertas principales en un adarve que esta cerca de la dicha puerta (judería) a la mano izquierda como entran por la dicha puerta de la juderia cerca del dicho monasterio de San Juan de los Reyes las cuales dichas casas las tiene ... johan Jarada el viejo mercader de toledo por si y por sancho y pedro sus hijos e hija francisca de la fuente su mujer que dios aya. Hija de Sancho gonzalez procurador por $200 \mathrm{mrs}$ de tributo... Las cuales dichas casas alindan con otras casas del dicho Sancho gonzalez procurador que dicen las casas de atahona que son tributarias al hospital de la misericordia y de la otra parte alindan con casa de Luis gonzalez mayordomo de Juan Gudiel que son de Juan Perez cuenca su nieto y de la otra parte con casa de los herederos de Pedro Franco hijo de Johan Gonzalez franco alguacil y a esta casas solian decir las casas de la higuera y la calle donde se deian la calle de los tintoreros y esta casa solia tener a tributo luis alonso mayordomo del dicho monasterio por los dichos $200 \mathrm{mrs}$ cada año ... fizo reconocimiento el dicho tributo al monasterio mencia gonzalez mujer de Sancho Gonzalez por su en nombre de Johan de Francisca su hijo. 1477. Una casa que el barrio que solia ser juderia cerca de los tintoreros que dicen las casas de la Figuera.» Documento transcrito por A. YUSTE.

21 A.S.D.R., libro 3, doc. 16, CD-Rom (Real Fundación de Toledo 2002).

22 A.S.D.R., Becerro de Santo Domingo el Real, 1802. Los reconocimientos de tributos durante el siglo XVI son los siguientes: Juan de Jarada en 1507, Sancho de la Fuente en 1524, Juan de la Fuente en 1561, Pedro González de las Zayas en 1585. En un documento de 1524, A.H.N. 7239, Sancho de la Fuente se refiere a «Juan de Jarada mercador». 
situación del inmueble, cuyo nombre no se vuelve a mencionar en los documentos ${ }^{23}$. No obstante, el nombre de algunos de sus posteriores ocupantes se encuentra en los registros de una casa a la que se llama «del arquillo» ${ }^{24}$. En 1638, Luis de Zayas reconoce el censo de 200 maravedís sobre la casa, situada «a la puerta de la lena (leña) de San Juan de los Reyes». Consta en algunos reconocimientos de tributos que ésta se encuentra, diez años después, «frontero a la caballeriza de San Juan de los Reyes», y al cabo de otros cien años, «frente a la porteria de San Juan de los Reyes». La casa de la Higuera ya no aparece en los documentos del monasterio de Santo Domingo el Real después de 1780.

\section{INTERPRETACIÓN Y DISCUSIÓN}

\section{La sinagoga del Sofer, la casa de la Higuera y la de la Atahona} a mediados del siglo $\mathrm{XV}$

De la lectura de los documentos anteriormente expuestos se desprende que don Çulemán Jarada poseía en 1391 una casa conocida como de la Higuera, entre la casa de la Atahona y la sinagoga del Sofer. La sinagoga del Sofer, la casa de la Higuera y la casa de la Atahona formaban un conjunto que nosotros hemos restituido ${ }^{25}$ (Figura 1) partiendo de las medidas y descripciones de 1455 y 1460. En la segunda mitad del siglo XV, concretamente en 1460, la casa de la Higuera presenta dos puertas: la principal, calle abajo, abre «a la

\footnotetext{
23 A esta casa se la llama «de la Higuera» en 1507 , pero a partir de esta fecha ya no se le aplica este nombre.

${ }^{24}$ Después de haber sido confiada a los herederos de la mujer de Sancho de la Fuente, la casa de la Higuera fue habitada después de 1585 por Pedro González de Zayas, cuyo nombre aparece como el de uno de los vecinos de «la casa del arquillo qre fue bariunia y dos apocentos alto de los herederos de juan de Cueva alinda casa de Juan de Cordova y otra de Pedro Gonzalez de Zayas y espaldas mathias (maestro) Lopez de Andrada tienda de un barbero».

${ }^{25}$ Hemos llevado a cabo la restitución basándonos en la descripción de la casa o. upada por Sancho González, realizada en 1455, incluida en los archivos del hospital de la Misericordia (A.D.P.T., Hospital de la Misericordia, Libro 1, 1455).
} 
juderia ${ }^{26} \gg ;$ la de arriba «sale a la calle principal» (Figura 5). El inmueble se imbrica en una casa «que eran xinoga» y ofrece una puerta al nivel del entresuelo que da a la casa de la Atahona ${ }^{27}$. Esta última, que «linda de una parte con casa de la Higuera... y de la otra la xinoga que disen del sofer y de la otra herederos de ferran gonzalez y calle publica real», presenta también dos puertas, una de ellas «una puerta que sale a la calle ${ }^{28}$ ». A partir de la segunda mitad del siglo XV, la casa de la Higuera y la de la Atahona tributan cada una 200 maravedís al monasterio de Santo Domingo el Real y 260 maravedís al hospital de la Misericordia. La sinagoga del Sofer dejó probablemente de ser utilizada como tal en 1391 tras los tumultos y asaltos de que fueron víctimas los judíos y que provocó la marcha de don Çulemán. No sabemos qué fue de ella, pues no se la vuelve a mencionar desde 1480. Conforme a una orden de los Reyes Católicos, no podía ser puesta en venta ni ocupada tras la expulsión de los judíos ${ }^{29}$. Por el contrario, los documentos nos permiten saber qué fue de la Atahona hasta 1509 y de la casa de la Higuera hasta 1780 .

\section{LA CASA DE LA HigUERA}

Seguiremos a los ocupantes de la casa de la Higuera y las descripciones sucesivas de ésta a fin de situar el conjunto anteriormente restituido.

En 1391, la casa de la Higuera se sitúa «en la que solia ser juderia» y «cerca de los tintores»; en 1402 «en la que fue juderia»; en

\footnotetext{
${ }^{26}$ Probablemente a la calle que se llamaba a fines del siglo XIV «calle de Abendonja» (véase F. CANTERA Burgos, «Relieves históricos de la judería de Toledo»).

${ }^{27}$ El texto del libro de las medidas de la casa figura en el anexo.

28 A.D.P.T., Hospital de la Misericordia, libro 1, 1455.

29 A.H.N., cajón 10, resumen en P. LEÓN TELlo, Judíos de Toledo, II, pág. 573, n. ${ }^{\circ}$ 1604: «1492, junio, 25, Guadalupe. Los Reyes Católicos mandan que, al salir los judíos de estos reinos, no vendan las sinagogas, censos y posesiones comunes, y que nadie se las compre, bajo la pena de perdimiento.»
} 
1403 «en la juderia»; en 1460 «a los tintores cerca de la puerta de la juderia». Se localiza, pues, en el barrio judío, pero las referencias al ámbito de éste carecen de precisión. Es importante seguir otra vía para concretar la ubicación probable del inmueble que nos ocupa.

En documentos posteriores a 1477 , leemos que esta casa se eleva «cerca de la puerta de la juderia a la mano izquierda como entran por la puerta de la juderia cerca del monasterio de San Juan de los Reyes ${ }^{30} \gg$. Se precisa que su puerta se encuentra sucesivamente frente a «la puerta de la lena» del monasterio de San Juan de los Reyes en 1638, «frontero a la caballeriza de San Juan de los Reyes» en 1648, «frontero a la porteria de los bastimientos del monasterio de San Juan de los Reyes» en 1730, y «frente a la porteria de San Juan de los Reyes» en 1748. Dicho de otra manera, los alrededores inmediatos de la casa de la Higuera cambiaron en el último cuarto del siglo $\mathrm{XV}$, en el curso del cual comienza la construcción del monasterio, en parte sobre el solar de casas que se fueron adquiriendo desde $1477^{31}$. Entre 1477 y 1748 el monasterio sufrirá profundas transformaciones, en particular la construcción de un segundo claustro junto al primero entre 1526 y $1534{ }^{32}$. Es importante, por lo tanto, precisar la situación de la puerta de San Juan de los Reyes a la que se refieren los textos citados de los siglos XVI y XVIII. Con este propósito nos referiremos a otras casas del mismo barrio.

El monasterio de San Pedro Mártir percibía en 1562 un tributo de 300 maravedís sobre una casa «en la perrochia de santo tome a san Juan de los Reyes» que lindaba «por tres partes con tres calles reales e por otra parte con el cobertizo del capellan mayor» y presentaba una fachada «enfrente de la porteria de san Juan de los

\footnotetext{
30 «A fines del siglo XV, hacia 1495-96, se trabajaba en el claustro principal del monasterio, que todavía no estaba acabado.»; véase F. MARÍAS, La arquitectura del renacimiento en Toledo (1541-1631), III (Toledo 1986) págs. 86-92.

${ }^{31}$ Véase nota 15.

${ }^{32}$ F. MARÍAS, La arquitectura del renacimiento en Toledo: «El segundo claustro de la casa o cuarto del Rey fue construido en época de Carlos V, seguramente después de otorgada la licencia de 1526 y antes de 1534 , aunque no se menciona de forma explícita esta obra.»
} 
Reyes ${ }^{33}{ }$. Las medidas de esta casa permiten identificarla con el actual n. ${ }^{\circ} 9$ de la calle de San Juan de los Reyes ${ }^{34}$, cuyo entorno conserva aún rasgos del siglo XVI ${ }^{35}$. La fachada de la casa del monasterio de San Pedro Mártir y los dos adarves que la encuadran se encontraban frente a «la porteria de San Juan de los Reyes». Planteamos la hipótesis de que el adarve al oeste de esta casa no es otro que «el callejon enfrente de las cadenas de la porteria del monasterio de San Juan de los Reyes», al fondo del cual se alzaba una casa principal, tributaria en 1592 de 1000 maravedís al monasterio de Santa Clara ${ }^{36}$. El documento que da cuenta de este censo indica que la casa linda con la de don Juan de Silva, conde de Portalegre. Esta última casa, que figura en el plano del Greco ${ }^{37}$, sirve de referencia para la localización del adarve y de las casas de San Pedro Mártir y de Santa Clara. De este modo podemos identificar las casas que se dicen situadas frente a la portería del primer claustro ${ }^{38}$; no se trata ni de la Atahona ni de la casa de la Higuera, que hemos de situar frente a la otra entrada del monasterio señalada en el segundo claustro ${ }^{39}$ (Figura 2). La puerta de servicio del monasterio de San Juan de los Reyes, situada en el recinto del segundo claustro, figuraba en el plano del monasterio realizado en $1580^{40}$. Conducía a diversos lugares de servicio del citado claustro,

33 Véase nota 15.

${ }^{34}$ Una viga con inscripciones cúficas, encontrada en uno de los muros de esta casa, fue enviada en 2002 al Museo Sefardí por José Aguado, a quien agradecemos aquí la ayuda que nos ha prestado.

${ }_{35}$ Junto al muro oeste persiste un adarve abierto, cuyo acceso está protegido por una verja; en el muro este, la casa limítrofe presenta un espacio largo y estrecho, indicio de un adarve privatizado.

${ }^{36}$ A.H.P.T., Becerro de Santa Clara.

37 Plano del Greco, 1606-1614.

${ }^{38} \mathrm{La}$ entrada lateral fue reemplazada por otra frontal ya en el siglo XX debido al ensanchamiento de la calle de San Juan de los Reyes.

${ }^{39}$ Esta puerta ha visto cambiar su denominación a lo largo del tiempo: se la ha llamado sucesivamente «puerta de la leña», «de la caballeriza» y «de los bastimientos».

${ }^{40}$ A.H.N., Inquisición, carp. 9 , n. ${ }^{\circ} 112$, leg. 3079. En este plano de la planta baja, realizado por Nicolás de Vergara, figura en el extremo opuesto a la iglesia una "puerta del sitio de los servicios de criados», que indica la entrada de los servicios en la planta sótano, cuyo levantamiento no figura en el plano del siglo XVI. 
que persisten en parte en los sótanos y la sala de exposiciones de la actual Escuela de Artes ${ }^{41}$.

\section{CONCLUSIÓN}

La demostración precedente nos lleva a considerar que el conjunto (Figura 3 ) formado a fines del siglo XV por la casa de la Higuera, la de la Atahona y la antigua sinagoga del Sofer se elevaba en una manzana, destruida en $1986^{42}$, situada entre la calle llamada «calle principal», que descendía de la iglesia de Santo Tomé a la «puerta de la juderia» (actual calle del Ángel) y la calle de San Juan de los Reyes frente a unas casas en cuyo emplazamiento se levantaría en el siglo XVI el segundo claustro de San Juan de los Reyes. Esta propuesta conlleva algunas puntualizaciones sobre la judería, cuyos límites no se han llegado a describir de manera precisa, como hemos dicho más arriba, y sobre la sinagoga del Sofer.

En lo que concierne a la judería, subrayemos en primer lugar que la «calle principal», a la que daba la puerta alta de la casa de la Higuera, conocida en el siglo XVII como la calle que desciende «del arquillo para San Juan de los Reyes», estaba fuera de la judería. Por otra parte, como la casa de la Higuera se alzaba nada más entrar en la judería, por la puerta de ésta a la izquierda, y la Atahona estaba «fondon de la puerta de la juderia de parte de fuera», el contacto entre estas dos casas coincidía con el límite entre la judería y el espacio exterior. Se desprende de esto una propuesta de situación de

${ }^{41}$ Véase F. MARÍAS, La arquitectura del renacimiento en Toledo, III, pág. 88, que nos recuerda que el segundo claustro de San Juan de los Reyes, que se mantenía en ruinas desde el incendio del monasterio durante la Guerra de la Independencia, fue incorporado a la Escuela de Artes.

${ }^{42}$ A.M.T., demolición del inmueble $\mathrm{n}^{\circ} 23$ calle del Ángel y $\mathrm{n}^{\circ} 6$ calle Reyes Católicos, 1984, expediente 191/84, y C. Delgado VAlero, Arte hispano-musulmán (artículos) (Madrid 2001) págs. 495-509. Las excavaciones de urgencia realizadas cuando la manzana ya se había derruido revelaron la existencia de un pozo y dos aljibes excavados en la roca en la esquina de las calles del Ángel y Reyes Católicos, así como de una fuente y dos aljibes en ladrillo al sur de la misma manzana. 
la puerta y del límite de la judería en esta área, que materializa la Figura 4. Debemos traer a colación otro hecho. La existencia de una fuente permanente algo más arriba de la casa de la Higuera hace plausible la presencia de tintoreros, al principio del siglo XV, en las cercanías de la puerta de la judería. Este manantial, que se mantiene todavía en una casa llamada «de la fuente», alimentaba una canalización de ladrillo que, descendiendo en dirección del «degolladero» por la sinagoga del Sofer, seguía al este del segundo claustro (Figura 4) ${ }^{43}$.

A propósito de la sinagoga del Sofer, de la que sólo tenemos noticias pertenecientes a la época en que ya estaba abandonada, insistimos en que se situaba cerca de una puerta de la judería, lo que debió de conferirle un papel importante. El muro correspondiente, que existía aún en el siglo XVI frente al segundo claustro de San Juan de los Reyes, como muestra el plano del Greco, desapareció en la segunda mitad del siglo XIX. Su huella se manifiesta como una plaza cuadrangular, en 1858, en el plano de Coello. El muro sin duda se había derrumbado. Nos sentimos tentados de atribuir a la sinagoga del Sofer el capitel y la viga encontrados enfrente de ella, al otro lado de la calle entre los escombros del segundo claustro, el capitel hacia $1873^{44}$ y la viga con posterioridad a esta fecha. Amador de los Ríos pensaba que el pequeño capitel, que tiene inscripciones hebraicas y árabes, pudo pertenecer a una sinagoga que no era Santa María la Blanca. F. Cantera ${ }^{45}$ lo dató en la segunda mitad del siglo XII o principios del XIII. En cuanto a la viga mudéjar, fue datada de 1180 por Zelson ${ }^{46}$, que opinaba que había servido de dintel de la puerta de una sinagoga sin duda importante, como era probablemente la del Sofer.

\footnotetext{
${ }^{43}$ Esta canalización en ladrillo llega, en el actual jardín de la Escuela de Artes, a un registro profundamente enterrado, donde converge otra canalización.

${ }^{44}$ R. AMADOR DE los Ríos Y VILlalta, Monumentos arquitectónicos de España (Toledo - Madrid 1905) págs. 481-486.

45 F. CANTERA BuRgos, «Inscripciones hebraicas en Toledo», Sefarad 4 (1944) págs. 45-72; F. CANTERA Burgos, «Epigrafía hebraica en el Museo Arqueológico de Madrid», Sefarad 11 (1951) págs. 105-111.

${ }^{46}$ L. G. ZELSON, «Viga mudéjar con inscripción hebraica en Toledo», Boletín de la Real Academia de la Historia 89/2 (1926) págs. 318-321; F. CANTERA Burgos y J. M. Millás VAllicrosa, Las inscripciones hebraicas de España (Madrid 1956) n. ${ }^{\circ} 228-229$, págs. 332-335.
} 
En conclusión, retendremos que el estudio detallado de los documentos del monasterio de Santo Domingo el Real y del hospital de la Misericordia, y el análisis de lo edificado permiten situar con precisión, en la ciudad medieval, el conjunto formado por la sinagoga del Sofer, la casa de la Higuera y la de la Atahona, de las que proponemos una representación (Figura 4). Por otro lado, añaden luz a nuestros conocimientos sobre el barrio de la «juderia vieja», que se convertiría en el siglo XV en el «barrio nuevo». Nuestro desconocimiento sobre este último punto es grande todavía. Esperamos volver sobre él más adelante. La sinagoga del Sofer, probablemente construida en la segunda mitad del siglo XII o a principios del XIII, habría dejado de servir de lugar de culto en 1391.

\section{RESUMEN}

A partir de la consulta de una variada evidencia documental de los siglos XIV a XVI, así como de la reconstrucción de la urbanística $\mathrm{dZ}<$ el barrio judío medieval de Toledo, el autor logra identificar la sinagoga llamada «del sofer» (principios del siglo XIII (?) hasta 1391, cuando deja de ser utilizada como tal), hoy desaparecida. Así mismo, realiza una propuesta de localización precisa junto a la «puerta de la judería» y la calle principal de ésta, enfrente del actual monasterio de San Juan de los Reyes.

PALABRAS ClAVE: sinagoga, barrio judío, Toledo, historia de los judíos.

\section{SUMMARY}

Through the analysis of a varied documentary evidence from the late $14^{\text {th }}$ up to the $16^{\text {th }}$ centuries, and the reconstruction of the medieval Jewish quarter of Toledo, the author identifies the so-called "sinagoga del sofer» (early $13^{\text {th }}$ century [?] up to 1391), today disappeared. A proposal is made to locate the lost building near the «puerta de la judería» and the main street of the Jewish quarter, just opposite the monastery San Juan de los Reyes.

KEYWORDS: synagogue, Jewish quarter, Toledo, History of the Jews. 


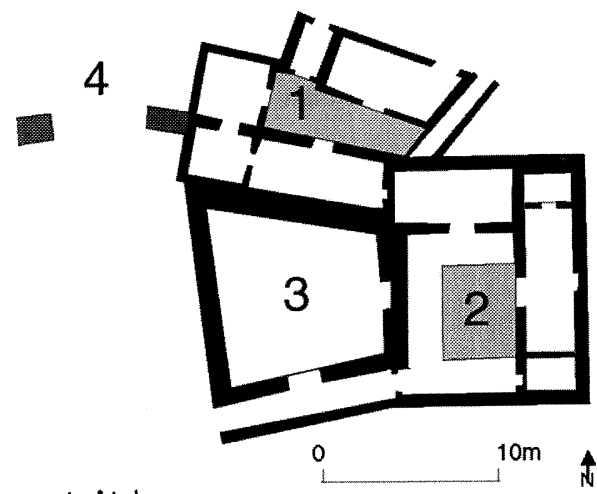

1. Atahona

2. Casa de la Higuera

3. La Sinagoga del Sofer

4. Puerta de la Judería

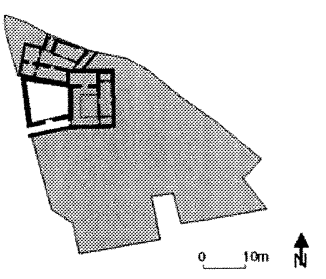

Situación en la manzana

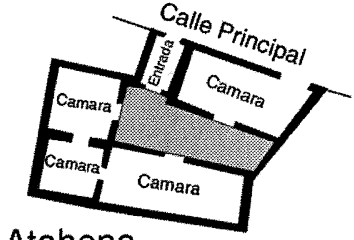

1. Atahona
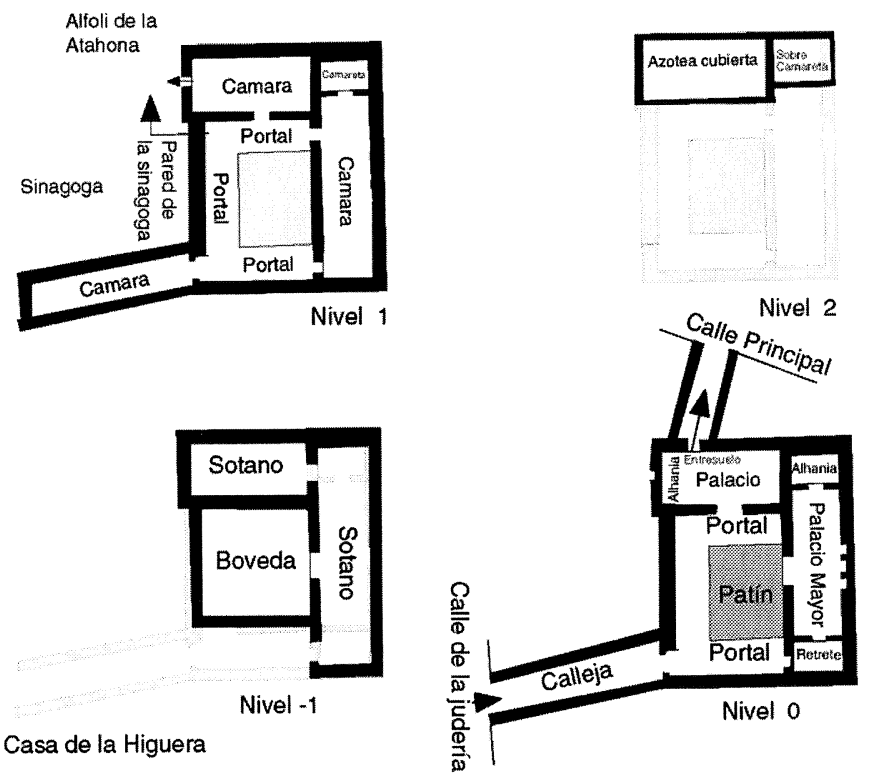

2. Casa de la Higuera

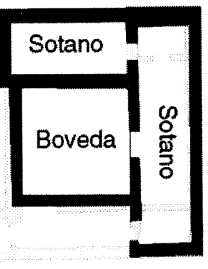

Nivel -1

Fig. 1. Sinagoga del Sofer, Atahona, casa de la Higuera en 1460: restitución del plano. 


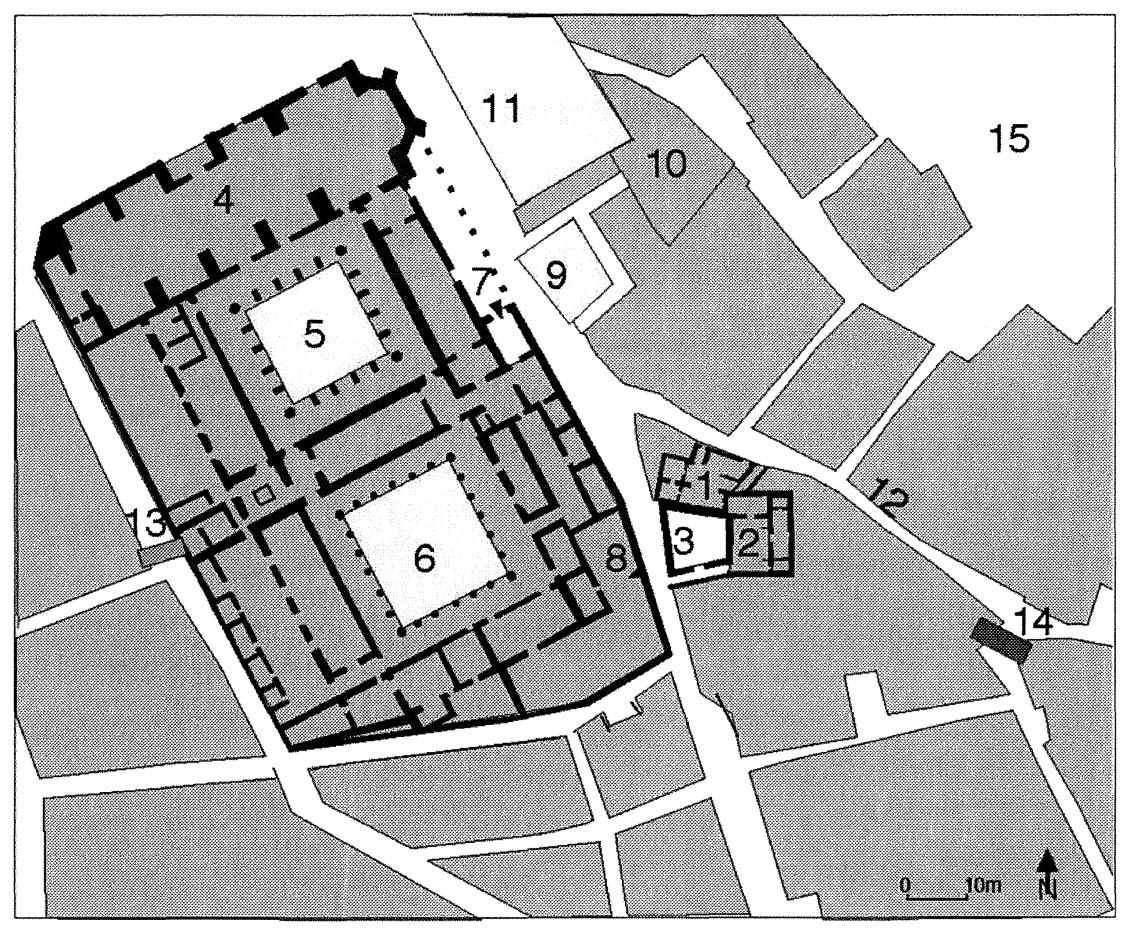

Fig. 2. El conjunto de la casa de la Higuera frente al monasterio de San Juan de los Reyes hacia 1580.

Conjunto de la casa de la Higuera: 1. Atahona; 2. Casa de la Higuera; 3. Sinagoga del Sofer.

Monasterio de San Juan de los Reyes: 4. Iglesia; 5. Primer claustro (siglo XV); 6. Segundo claustro (siglo XVI); 7. Portería; 8. Puerta de servicio.

Casas frente a la portería: 9. Casa tributaria de San Pedro Mártir; 10. Casa tributaria de Santa Clara; 11. Casa principal del Conde de Portalegre; 12. Calle de Santo Tomé a la puerta del Cambrón; 13. Cobertizo Calle del Mármol; 14. Arquillo de la Judería; 15. Barrio del Alacava. 


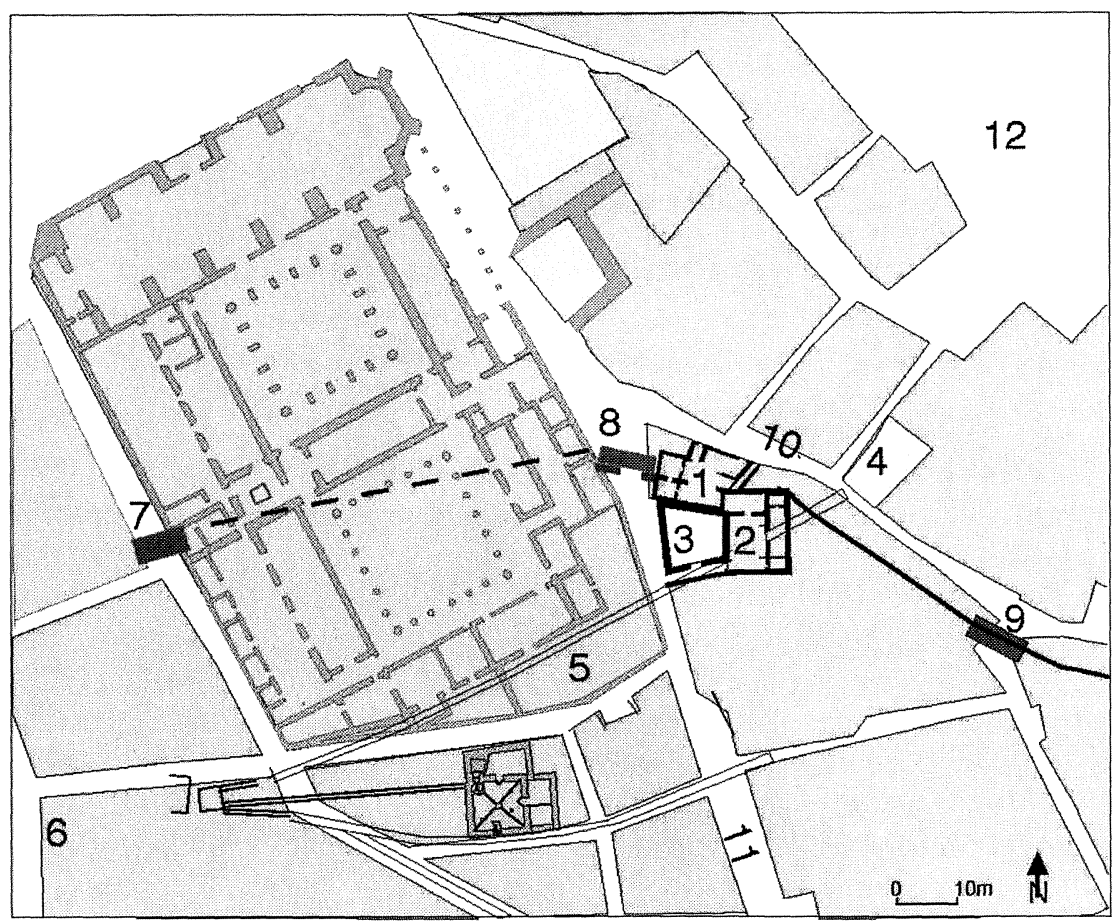

Fig. 3. El conjunto de la Sinagoga del Sofer hacia 1460.

1. Atahona; 2. Casa de la Higuera; 3. Sinagoga del Sofer; 4. Casa de la Fuente; 5. Barrio de los Tintoreros; 6. Degolladero; 7. Cobertizo de la Calle del Mármol; 8. Puerta de la Judería; 9. Arquillo de la Judería; 10. Calle principal de Santo Tomé a la puerta del Cambrón (calle de Abendonja); 11. Calle de la Judería; 12. Barrio del Alacava.

Cerca de la Judería : trazado hipotético Conducto de agua 


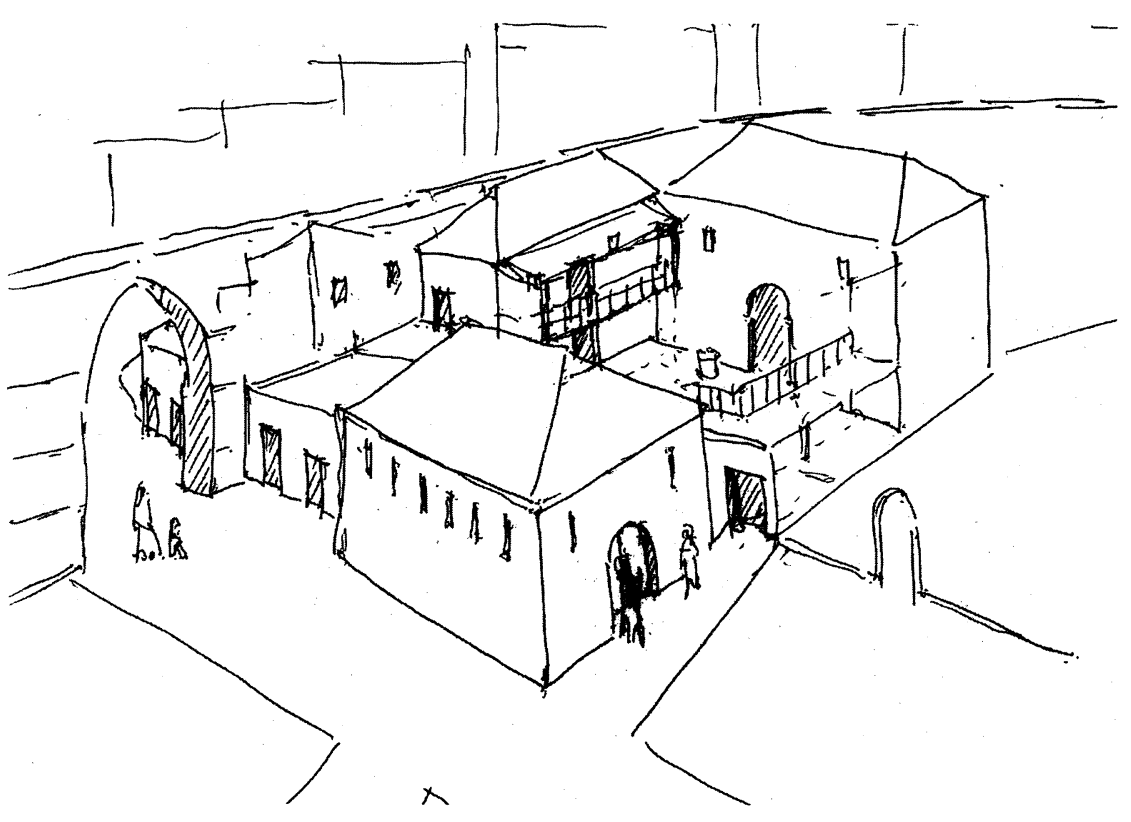

Fig. 4. Restitución de la Sinagoga del Sofer en el siglo XIV.

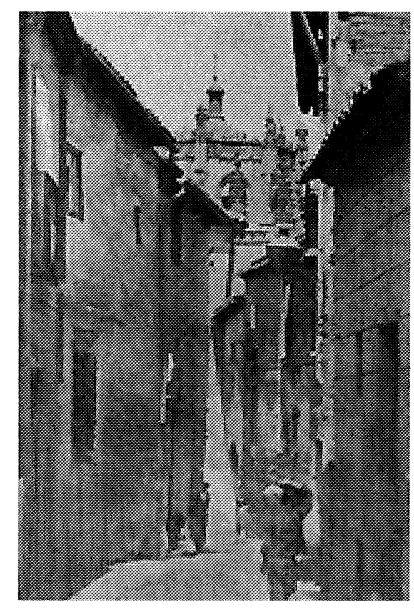

Fig. 5. La puerta de la casa de la Higuera ¿que sale a la calle principal? (calle del Ángel), segunda mitad del siglo XIX, hoy desaparecida. Colección privada: Luis Alba. 


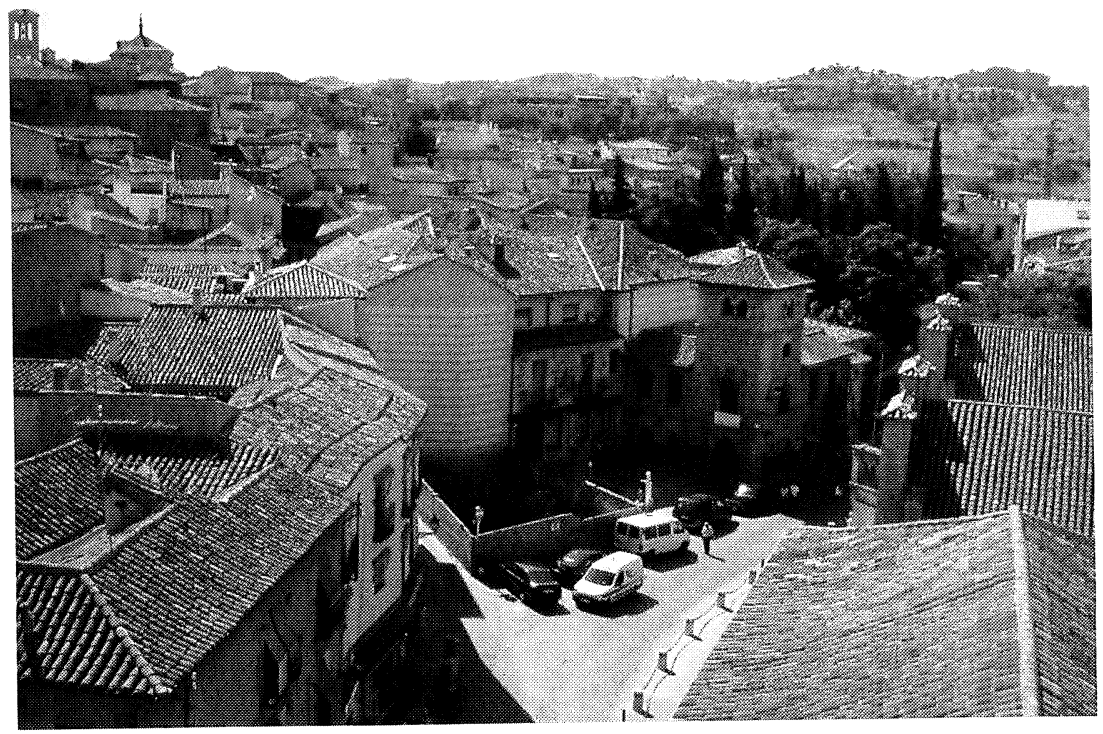

Fig. 6. El sitio de la antigua sinagoga del Sofer, 2002. 